\title{
Fosbretabulin Tromethamine
}

National Cancer Institute

\section{Source}

National Cancer Institute. Fosbretabulin Tromethamine. NCI Thesaurus. Code C83721.

The tromethamine salt form of prodrug fosbretabulin, a water-soluble phosphate derivative of a stilbenoid phenol derived from the African bush willow (Combretum caffrum) with antineoplastic activities. Upon administration, fosbretabulin is dephosphorylated to its active metabolite, combretastatin A4, which targets and binds to tubulin dimers and prevents microtubule polymerization, thereby resulting in mitotic arrest and apoptosis in endothelial cells. As apoptotic endothelial cells detach from their substrata, tumor blood vessels collapse; the acute disruption of tumor blood flow may result in tumor necrosis. 